\title{
MICROSCOPIC ASPECTS OF FRICTION
}

\author{
S. CIRACI and A. BULDUM \\ Department of Physics, Bilkent University \\ Bilkent 06533 Ankara Turkey
}

\begin{abstract}
In this work we first present an analysis of the interaction energy and short range forces between two parallel and atomically flat metal slabs. Using the self-consistent field pseudopotential method within the local density approximation we obtain the interaction energy surface and lateral force variation for two parallel metal slabs in relative motion under a constant loading force. Based on these results we investigate the stick-slip motion in the dry sliding friction. The effects of material parameters and loading force on the friction force are analyzed by using an infinite chain of atoms having two degrees of freedom in the lateral and normal directions. The foreign atoms between two sliding surfaces prevent them from strong adhesive contact and wear. This is the boundary lubrication and takes place under the low coverage of inert gas atoms $(\Theta<1)$. In order to reveal the essential features of stick-slip motion of the lubricant atoms and the energy damping thereof, we finally studied the motion of Xe atom on the metal surface manipulated by a metal tip.
\end{abstract}

\section{Introduction}

An object moving at the close proximity to another one induces nonconservative forces, which cause to the dissipation of energy of motion. This phenomenon is known as friction [1-4]; the tribology is the science which deals with friction. The friction is involved in many fields ranging from earthquakes to molecular collisions. Not only its importance in technology, but also underlying complex physics makes the friction very attractive area for researchers. The short and long-range interaction occurring between two objects induce the friction force. Atomic and frictional force microscope [5-7], which achieved nanonewton precision in measuring those forces have made great impact in tribology. Experiments [8-10] providing precise data together with model calculations [11-16] and large scale computations [17-19] have contributed for better understanding of various phenomena in friction.

In order to reduce the friction and wear lubricants are introduced between two moving objects. Molecules or foreign atoms ( such as Xe) which are introduced between two surfaces in relative motion prevent the surfaces from adhesive contact. The dry sliding friction between atomically flat ( commensurate or incommensurate) sliding surfaces is perhaps the simplest, but at the same time most fundamental type of friction in tribology. It may involve several interesting, atomic scale phenomena. Such as adhesion, wetting and atom transfer, strain induced phase transition, local surface reconstruction. The dissipation of 
energy is the most complex and interesting feature of friction. Non-equilibrium phonon and electron density created locally in the course of friction are dissipated by phonon-phonon and electron-phonon coupling. In the process of energy dissipation electron hole pairs as well as charge density waves can be excited [20-21].

Understanding the nature of nonconservative forces and various mechanisms of energy dissipation in friction requires detailed analysis of the atomic scale friction of surfaces with (and without) foreign atoms in between. In particular the variation of average friction force $\bar{F}_{f}$ with the normal loading force $F_{N}$ is important feature one would like to know. The effect of the material parameters ( such as the cohesive energy of surface atoms, elastic constants and surface structure etc ) are attracting interests. Apart from those phenomenological aspects, the microscopic theory of energy damping in friction is not well founded yet.

This work deals with the microscopic aspects of friction. We start with the analysis of the interactions and short and long-range forces between two objects. To study the stick-slip motion we consider the relative motion between two $\mathrm{Al}(001)$ slabs. Using ab-initio self-consistent field pseudopotential method we obtain the variation of the lateral force with the relative displacement, wherefrom the hysteresis curve and the average friction force are calculated. To investigate the effect of material parameters we use a one-dimensional infinite chain, in which atoms have degrees of freedom in the lateral and perpendicular directions. The elementary features of the boundary lubrication and the stick-slip type behavior are discussed in the tip induced lateral translation of Xe on a metal surface.

\section{Interactions and Forces Between Two Objects}

When two objects ( atoms, molecules, clusters or solid surfaces ) are close to each other the electrostatic repulsion is overcame by the rearrangement of electronic states. This leads to a net interaction (electrostatic plus exchange-correlation energy) at the expense of kinetic energy. The interaction energy $E_{i}(\vec{r})$ between two objects (say atomically flat $\mathrm{A}$ and $\mathrm{B}$ slabs) can be obtained by comparing the total energies of the individual slabs with the total energy of two combined slabs (A and $\mathrm{B}$ at close proximity) [22]. The relative position of the slabs is given by the vector $\vec{r}=\vec{r}(x, y, z)$ between two points $O_{A}$ and $O_{B}$ fixed in the slabs A and B, respectively. Here $z$ is the spacing between slabs A and B, and is perpendicular to the slab surfaces. For a given separation $z=z_{o}, E\left(x, y, z_{o}\right)$ has a two dimensional corrugation. The interaction energy between two parallel and flat surfaces vanish for $z=z_{c}(x, y)$, i.e. $E\left(x, y, z_{c}\right)=0$. The interaction is repulsive for $z<z_{c}(x, y)$ but attractive for $z>z_{c}(x, y)$. The attractive interaction is the adhesion between two objects. There is a continuous interplay between repulsive interaction and adhesion in the course of dry rolling friction, that involves friction and energy damping. The minimum of the interaction energy and the corresponding height $z=z_{e}(x, y)$ for a given lateral position $(x, y)$ is determined from $\left(\partial E_{i}(\vec{r}) / \partial z\right)_{x, y}=0$. The lowest value of $E_{i}$ (or highest adhesion energy) generally occurs when an atom of one commensurate surface (i.e $\mathrm{T}$ top site of $\mathrm{A}$ ) faces the hollow $(\mathrm{H})$ site of $\mathrm{B}$ at the other surface. An external normal force $F_{N}$ exerted to the object sets the separation $\bar{z}\left(x, y ; F_{N}\right)$ at a given lateral position $(x, y)$. If one of the commensurate $\operatorname{slab}(\mathrm{A})$ slides above (B) under $F_{N}, z=\bar{z}\left(x, y ; F_{N}\right)$ as well as $E_{i}(x, y, \bar{z})$ vary with the lateral displacement. The corrugation of interaction energy, $\Delta E_{i}(x, y, \bar{z})$, i.e 
the energy difference between the maximum and minimum value of $E_{i}$ along the lateral displacement is the most important parameter in friction. $\Delta E_{i}(x, y, \bar{z})$ varies with $F_{N}$, and may vanish for certain normal loading force. In some cases even it may become inverted beyond this value.

The force between A and B can be calculated either from the Hellman-Feynman theorem or from the derivative of $E_{i}(\vec{r})$. In this case, the lateral force corresponding to a fixed $F_{N}$ is given by [22]

$$
\vec{F}_{L}(x, y, \bar{z})=-\left\{\left[\frac{\partial E_{i}(\vec{r})}{\partial x}\right]_{z=\bar{z}} \vec{i}+\left[\frac{\partial E_{i}(\vec{r})}{\partial y}\right]_{z=\bar{z}} \vec{j}\right\}
$$

The variation of the perpendicular force is

$$
\vec{F}_{P}(x, y, z)=-\left(\frac{\partial E_{i}(\vec{r})}{\partial z}\right) \vec{k}
$$

The lateral and perpendicular force in Eq. (1) and Eq. (2) have electron-ion attraction and ion-ion repulsion, which essentially compensate each other almost completely at large separations. Since the electronic charge density around each nuclei is exponentially decaying $\vec{F}_{L}$ and $\vec{F}_{P}$ are short ranged.

Here we consider the interaction between two $\mathrm{Al}$ metals. Two infinite $\mathrm{Al}(001)$ slabs (A and B) are lattice matched and whole system display a two dimensional periodicity of the (001) surface of $\mathrm{Al}$ if the surface bravais lattice vectors of slabs are parallel. This situation allows us to use the periodic boundary condition and to adopt the momentum representation. For a given configuration one can expand the wave function on the plane waves, $\Psi_{n}(\vec{k}, r)=\sum a_{n}(\vec{k}+\vec{G}) \exp [i(\vec{k}+\vec{G}) \cdot \vec{r}]$ and carry out self-consistent field (SCF) calculation of the electronic structure and total energy within the local density approximation. Figure 1, illustrates the calculated variation of $F_{P}$ and $F_{L}$ between two $\mathrm{Al}(001)$ slab as a function $\overline{H T}$-line [23]. The lateral position of the top site, i.e $(x, y)$ relative to the bottom slab $\mathrm{B}$ is shown in the inset. We note that at the symmetry point $\left(x_{s}, y_{s}\right)$ of the 2D unit cell $F_{L}\left(x_{s}, y_{s}, z\right)=0$, but it becomes significant at general points. For $(\mathrm{x}, \mathrm{y})$ at the $\mathrm{H}$ site $E_{i}$ as well as $F_{P}$ have largest magnitudes. The corrugation of interaction energy $\left(\Delta E_{i}\right)_{\text {maximum }} \simeq 0.5 \mathrm{eV} /$ atom occurs between $\mathrm{H}$ and $\mathrm{T}$ sites. The variation of $F_{L}(x, y, z)$ for $z=\bar{z}\left(x, y ; F_{N}\right)$ gives rise to stick-slip motion. While the stick stage of the motion is adiabatic and converts the kinetic energy into the potential (or elastic) energy, The slip stage is non-adiabatic. As a result the energy stored during the stick is damped by creating local, non-equilibrium phonon density. Sliding object normally slips less than a lattice parameter, but at some certain circumstances it can slip more than a unit cell. Normally, $F_{L}$ and $F_{P}$ are conservative forces, but owing to the stick-slip motion the average of $F_{L}$ resists to the relative motion of $\mathrm{A}$ and $\mathrm{B}$ and becomes nonconservative.

The interaction between A and B involves also the Van der Waals forces (VdW). The origin of $\mathrm{VdW}$ force can be better described in terms of surface plasmons of $\mathrm{A}$ and $\mathrm{B}$, which are coupled through their electric field. Therefore the $\mathrm{VdW}$ force is long ranged and can occur even if the electronic states of separated electrodes are decoupled and hence the exponentially decaying short-range forces are negligible. Although the continuum description leaves out the discrete nature of the tip, the generalized Liftshitz approach is, nevertheless convenient for metal electrodes. In the present case, where A and B are parallel the electric field of a plasmon mode with a wave vector $\vec{k}_{\|}$varies with $\exp \left(-k_{\|} z\right)$ and hence, 


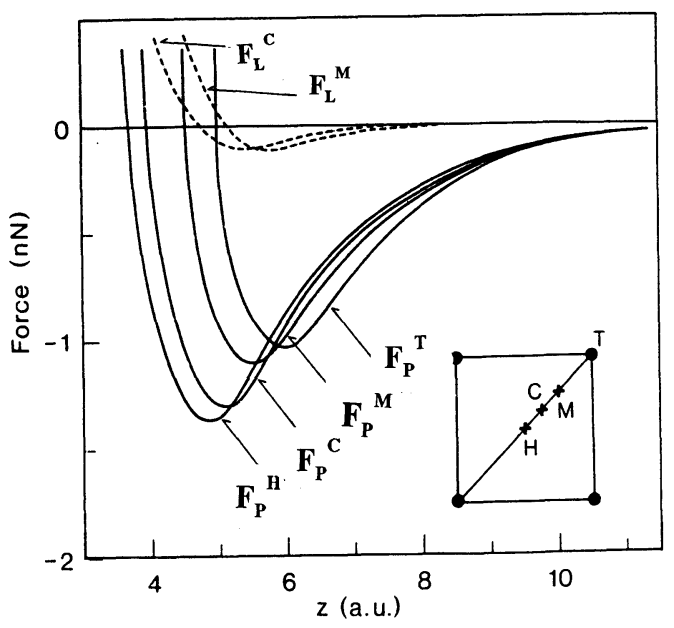

Figure 1: Lateral $\left(F_{L}\right)$ and perpendicular $\left(F_{P}\right)$ short-range forces versus separation $z$ between two $\mathrm{Al}(001)$ slabs calculated by using self-consistent pseudopotential method within local density approximation. The inset shows the primitive unit cell of $\mathrm{Al}(001)$ surface with hollow $(\mathrm{H})$, top $(\mathrm{T})$ sites along $\overrightarrow{H T}$-diagonal.

the VdW interaction is dominated by the long-range wavelength modes at large $z$. Existing treatments rely on summing the asymptotic interaction law $-\mathrm{Cr}^{-2}$ over the volume of the interacting slabs, which are valid only for sufficiently large separations. The VdW interaction between the atoms in the slabs A and B are weak. However, owing to their large range the forces on atoms further away can be approximated by an integral over body forces. This gives a relatively strong but essentially uncorrugated attraction. Consequently, long-range interaction does not contribute directly to the corrugation of $F_{L}$, except that it affects the magnitude of the normal force. As $z \rightarrow 0$ the Liftshitz formula diverges, so it can not be adequate to represent the $\mathrm{VdW}$ interaction for small $z$. The correct description of the $\mathrm{VdW}$ then requires the proper account of the collective behavior of electrons with nonlocal microscopic dielectric theory.

\section{Ab initio Calculations of Atomic Scale Friction}

The interaction energy surface can be constructed by calculating the minimum of $E_{i}(x, y, z)$ at each grid point $(x, y)$ in the surface unit cell. Note that the $(x, y)$ position of a surface atom in A scans the unit cell of the slab B. Here we obtained the interaction energy surface $E_{i}(x=y, \bar{z})$ corresponding to a loading force, $F_{N}$ by extrapolating $F_{P}(z)$ in Fig.1. The variation of the lateral force $F_{L}\left[x, y, \bar{z}\left(x, y ; F_{N}\right)\right]$ are calculated according to Eq.1 to study atomic scale friction between two $\mathrm{Al}(001)$ slabs. Note, however, that $\mathrm{A}$ facing the $\mathrm{H}$-site of $\mathrm{B}$ at $z=z_{e}$ (i.e $F_{N}=0$ ) makes a single perfect slab consisting of $\mathrm{A}$ and $\mathrm{B}$. One can only distinguish two different slabs if $\mathrm{A}$ deviates laterally from the $\mathrm{H}$-site of $\mathrm{B}$ or $z \neq z_{e}$. Foreign atoms with coverage $\Theta \sim 1$ also separate $\mathrm{A}$ and $\mathrm{B}$. In order to explore the friction between two slabs we nevertheless consider $\mathrm{A}$ and $\mathrm{B}$ as two different objects in relative motion. The variation of $F_{L}$ along the $\overline{H T}$-line at a given $F_{N}$ (or the corrugation of the lateral force 

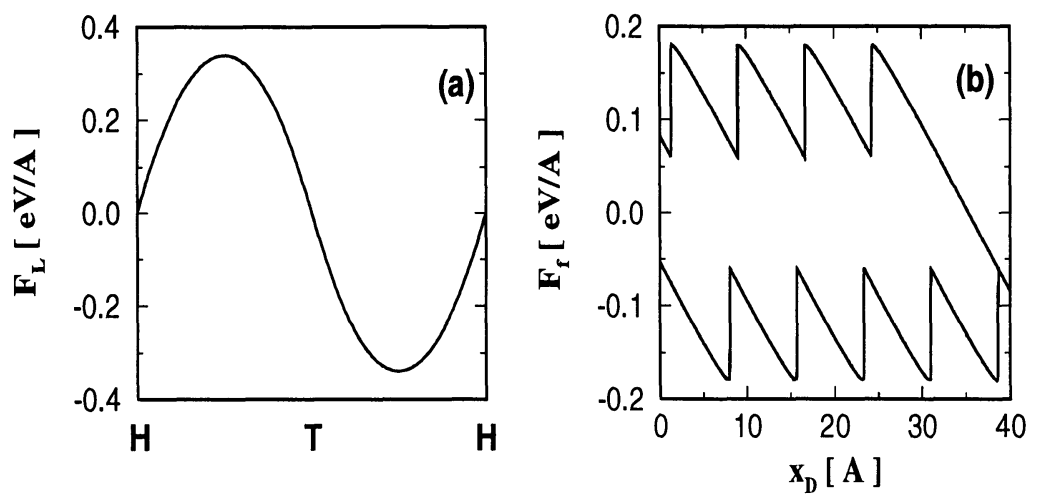

Figure 2: (a) Variation of the lateral force $F_{L}\left(l ; F_{N}=0\right)$ with the displacement $l=\left(x^{2}+\right.$ $\left.y^{2}\right)^{1 / 2}$ along $\overline{H T}$-line (b) The hysteresis curve corresponding to the stick-slip motion of $\mathrm{Al}(001)$. The effective force constant of the moving agent is $k_{t}=0.02 \mathrm{eV} / \AA^{2}$

$\left.\Delta F_{L}\right)$ is essential for friction if the effective force constant in the direction of motion is soft enough to lead to bistable solutions. Figure 2 illustrates the calculated [23] of corrugation of $F_{L}$ and the friction force $F_{f}$ for the relative motion of $\mathrm{A}$ along the $\overrightarrow{H T}$. The average value of $F_{f}$ is finite and is against the motion of $\mathrm{A}$. By reversing the direction of motion one obtains the hysteresis curve in the stick-slip motion of two metal slabs calculated by an ab initio method. We note following features of ab initio calculations i) Under the normal and lateral forces the atoms on the surfaces of A and B undergo perpendicular and lateral displacements. The variation of $E_{i}$ and $F_{L}$ are modified due to the displacements of atoms. In the present and earlier studies of atomic scale friction such modification of $E_{i}(\vec{r})$ and $F_{L}(x, y, \bar{z})$ have not been taken into account. ii) It is assumed that the speed of displacement $v_{D}$ is much smaller than the speed of sound, and the process is adiabatic in the course of stick. This means that $W=\int \vec{F}_{L} \cdot d \vec{r}$ is equal to the increase of $E_{i}$. Furthermore, the energy stored in the system during the stick is released and is damped through the excitation of non-equilibrium phonons and other mechanisms of energy dissipation. iii) If the contact between surfaces occurs through asperities, small contact surfaces may experience not only relaxation but also local reconstruction, even wear. Depending upon the relative binding energies and the coordination numbers at the asperity, the atoms of one object can wet the other. iv) In the relative motion of large incommensurate or multigrain surfaces the corrugation of $E_{i}$ and $F_{L}$ diminish; the energy damping takes place through the stick-slip motion of individual atoms. As a result the hysteresis curve continues to exist, but the friction force becomes uniform.

\section{Model Calculations for Atomic Scale Friction}

Earlier Simple models such as Tomlinson [11], and Frenkel and Kontorova approaches [12] have been useful to study dry sliding friction. Our model, described in Fig. 3 aims to explore the effect of various material parameters on the atomic scale friction. In particular the stick-slip motion is investigated in the presence of local elastic deformation in the perpendicular direction. The moving (or sliding) object A is represented by a single atom. This can viewed as the apex atom of the tip in an atomic force microscope. Single atom is 


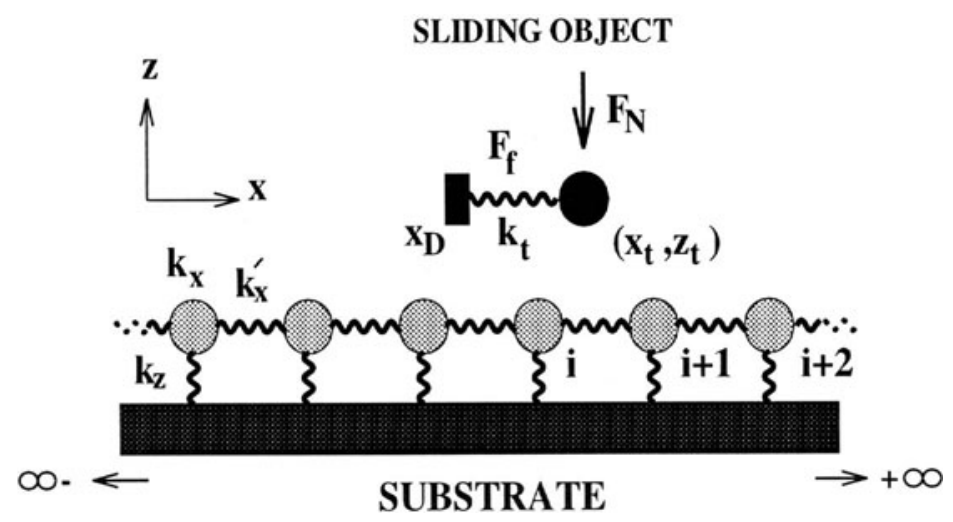

Figure 3: The model used to study the atomic-scale friction.

sensitive to the atomic structure and to the energy corrugation of the substrate, and has coordinates $\vec{\rho}_{t}\left(x_{t}, z_{t}\right)$. A sliding object incorporating several atoms could have contributed to the energy damping. This would be only a further elaboration of the present model. The agent that pushes the sliding object has coordinates $\vec{\rho}_{D}\left(x_{D}, z_{D}\right)$, where $z_{D}=z_{t}$. Note that the motion of atoms occurs in the lateral $(x)$ and perpendicular $(z)$ direction. The lateral force, acting on the sliding atom is given by $F_{L}=k_{t}\left(x_{t}-x_{D}\right.$ ) (assuming that $F_{L}=0$ at $\left.x_{D}=x_{t}\right)$. The interaction potential between the sliding atom and substrate atoms $V_{t i}\left(\left|\vec{\rho}_{t}-\vec{\rho}_{i}\right|\right)$ are represented by a Lennard-Jones pair potential having parameters $\epsilon=0.84$ $\mathrm{eV}$ and $r_{o}=2.56 \AA$. Those parameters are fitted to the physical properties of the Ni-metal. The substrate (B) is modeled by an infinite chain of atoms. This way various edge effects due to the finite size of the substrate are avoided. $\vec{\rho}_{i}\left(x_{i}, z_{i}\right)$ and $\vec{\rho}_{o i}\left(x_{i o}, z_{i o}\right)$ denote the equilibrium positions of the chain atoms with and without the moving object, respectively. The cohesion of the substrate is provided by the interchain potential. The substrate atoms are in their equilibrium positions, $\vec{\rho}_{o i}$ when $\sum_{i} V_{t i}=0$. The total potential of the whole system ( $\mathrm{A}+\mathrm{B}$ i.e sliding object and the substrate $)$ is given by

$$
\begin{aligned}
V_{T}= & \sum_{i} V_{t i}\left(\left|\vec{\rho}_{t}-\vec{\rho}_{i}\right|\right)+\frac{1}{2} k_{t}\left(x_{t}-x_{D}\right)^{2}+\sum_{i} \frac{1}{2} k_{x}\left(x_{i}-x_{i o}\right)^{2} \\
& +\sum_{i} \frac{1}{2} k_{x}^{\prime}\left[\left(x_{i+1}-x_{i+1, o}\right)-\left(x_{i}-x_{i, o}\right)\right]^{2}+\sum_{i} V_{p, i} \\
& +F_{N} z_{t}
\end{aligned}
$$

Here, $V_{p, i}$ is the potential of substrate atoms in the perpendicular direction; it is taken harmonic, i.e $V_{p, i}=k_{z}\left(z_{i}-z_{i o}\right)^{2} / 2$. The last term in $V_{T}$ indicates that an external perpendicular force $F_{N}$ is acting on the sliding atom. The total potential $V_{T}$ as expressed in Eq.(2) is reminiscent of the interaction energy discussed in the previous section. Here in $V_{T}$, the electronic contribution to interaction is robust. This corresponds to the normal force

$$
F_{N}=-\sum_{i}\left[\frac{\partial V_{t i}}{\partial z_{t}}\right]_{\overline{z_{t}}}
$$


and sets $z_{t}=\bar{z}_{t}$ for a given lateral position $x_{t}$. Then the equation of motion of the moving atom under the constant normal force can be easily written. The sliding atom applies a force to the substrate and induces local deformation. The strain energy stored in this local deformation spreads out in the substrate. This is the path of the energy transfer from the moving object to the substrate leading to the dissipation. The deformation propagates with the speed of sound $\sim 5000 \mathrm{~m} / \mathrm{s}$. In the present study we consider the low sliding speeds in the range of $v_{D} \sim 4 \AA / s$. This is relevant for AFM. Furthermore, we assume that the normal force $F_{N}$ does not cause to any kind of plastic deformation or wear; hence the energy which is transferred to the substrate by an induced local deformation spreads with the speed of sound, and is dissipated almost suddenly. These conditions justify the quasi-static approximation, in which the friction force $F_{f}$ at a given time $t$ is equal to the lateral force $F_{L}$. This requires the determination of the actual positions of substrate atoms $\left\{x_{i}, z_{i}\right\}$ for a given $x_{D}$ and $F_{N}$. Since all these coordinates are interrelated they can be calculated by using tedious iterative procedures. The interaction potential, $\sum_{i} V_{t i}$ involves 141 atoms at close proximity to the sliding object. Only 60 atoms out of 141 are allowed to relax under the interaction potentials. The rest of the substrate atoms which are far from the sliding object are kept in their original position since their displacements under the force exerted by the other atoms are insignificant. One $x_{D}$ at time $t$ is given we start by determining the equilibrium positions of the atoms for a given $\left(x_{t}, z_{t}\right)$ by using equilibrium conditions for the forces obtained from interaction potential. Note that the moving atom is under the forces $F_{N}, F_{L}$ and the force derived from interaction potential $\sum_{i} V_{t i}\left(\left|\vec{\rho}_{t}-\vec{\rho}_{i}\right|\right)$. In the iteration cycles $z_{t}$ is varied continuously to find $\bar{z}_{t}$, so that the normal force becomes equal to the desired value $F_{N}$. The lateral coordinate $x_{t}$ of the moving object is varied to balance $F_{L}$. Upon reaching the equilibrium condition at $x_{D}$, the friction force $F_{f}$ which is equal to $F_{L}$, is calculated from the actual values of $x_{D}$ and $x_{t}$. Increasing $x_{D}$ by $v_{D} \Delta t$ all steps of iterations are repeated to find $F_{f}$ for this new value of $x_{D}$. This way $F_{f}$ versus $x_{D}$ (or $F_{f}$ versus $t$ ) curves are obtained for right going or left going moving object. This leads to a hysteresis since the average of $F_{f}, \int_{x_{D 1}}^{x_{D 2}} F_{f} d x_{D} /\left(x_{D 2}-x_{D 1}\right)$ is finite and is equal to average friction force $F_{f}$. Using quasi-static approximation we calculated $F_{f}\left(x_{D}\right)$ and $\bar{F}_{f}$ and the hysteresis curve, i.e $F_{f}\left(x_{D}\right)$ curve in a cycle of $x_{D}$ and examined the effect of material parameters and $F_{N}$.

According to the definition of friction force in the present model the elasticity of sliding object $k_{t}$ (or the force constant of cantilever of AFM in lateral bending) is essential for the stick-slip motion and $F_{f}$. In fact, the lost of energy in the course of slip decreases as $k_{t}$ increases, and eventually the bistability leading to slip does not occur when $k_{t}$ exceeds the value set for a given substrate having the corrugation $F_{L}\left(x ; F_{N}\right)$. For a given $k_{t}$, the friction force $F_{f}$ is strongly dependent on the lateral and perpendicular elastic parameters of the substrate surface. The energy damping increases with decreasing lateral force constants. In general, the average energy damping increases with decreasing $k_{z}$ at a fixed $F_{N}$, and also with increasing $F_{N}$. However, $\bar{F}_{f}\left(k_{z}\right)$ at constant $F_{N}$ or $\bar{F}_{f}\left(F_{N}\right)$ at constant $k_{z}$ are not linear, and requires a detailed analysis. The essential aspects of nonlinearity, which were not taken into account before, are the deformation induced modification of the interaction potential and its anharmonicity. Present calculations show that the energy damping is large for small $k_{z}$ (i.e $\left.k_{z} \sim 5 \mathrm{eV} / \stackrel{\circ}{ }^{2}\right)$. $F_{f}$ increases with increasing $k_{z}$, passes through a minimum at $k_{z}=7.5 \mathrm{eV} / \AA^{2}$, and increases again with increasing $k_{z}$. Eventually it saturates 


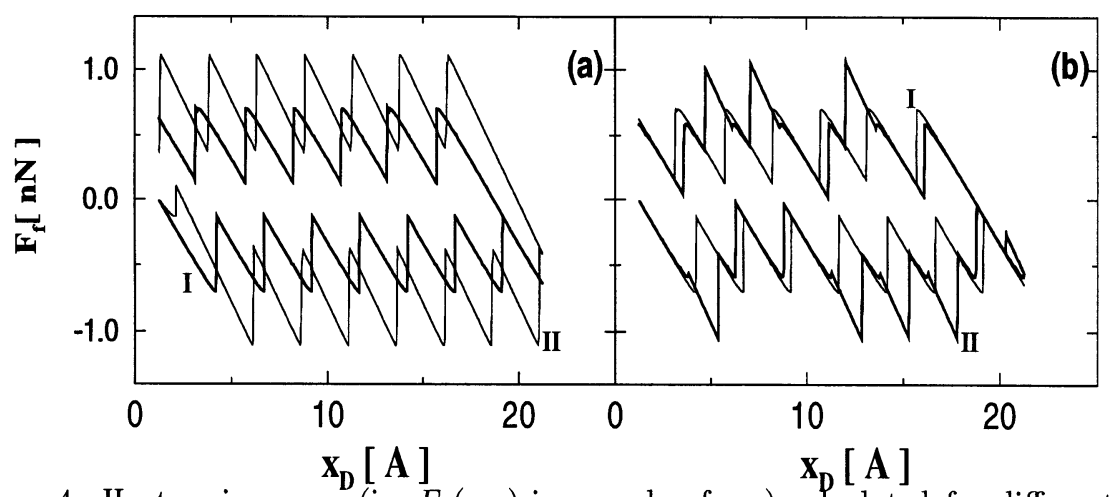

Figure 4: Hysteresis curves (i.e $F_{f}\left(x_{D}\right)$ in a cycle of $x_{D}$ ) calculated for different normal loading force. Elastic parameters are: $k_{t}=0.2, k_{x}=4.3, k_{x}^{\prime}=5.8, k_{z}=10 \mathrm{eV} / \AA^{2}$. (a) I: $F_{N}=0$, II: $F_{N}=1 \mathrm{nN}$. (b) I: $F_{N}=0$, II: $F_{N}=0.2 \mathrm{nN}$

as $k_{z} \rightarrow \infty$. In Fig. 4(a) and 4(b), the hysteresis curves for $F_{N}=0,0.2$, and $1 \mathrm{nN}$ at $\left(k_{x}=4.311 \mathrm{eV} / \AA^{2}, k_{x}^{\prime}=5.886 \mathrm{eV} / \AA^{2}, k_{z}=0.2 \mathrm{eV} / \AA^{2}\right)$ show that the energy damping increases with increasing $F_{N}$. It is also seen that the stick-slip motion becomes irregular for $F_{N} \neq 0$, and in the hysteresis curve two different state in stick-slip motion (or some kind of anisotropy) are distinguished. Such an effect disappears when the substrate surface becomes rigid in the perpendicular direction or the variation of $V_{T}$ in the perpendicular direction becomes strongly anharmonic. The origin of the anisotropy lies in the variation of $V_{T}$ with $F_{N}$ and hence in the deformation induced modification of $V_{T}$. Note that $V_{T}$ is low at the $\mathrm{H}$-site (between two substrate atoms, but increases at the T-site (on top of the substrate atom). The corrugation $\Delta V_{T}=V_{T}(T)-V_{T}(H)$ increases with increasing normal force for $k_{z} \gg 1$. On the other, $\Delta V_{T}$ varies with the normal force if the substrate surface is elastic (i.e $k_{z}$ is small ) in the perpendicular direction; $\Delta V_{T}$ is positive for $F_{N}=0$, but it decreases with increasing $F_{N}$ and eventually the corrugation is inverted at some range of $F_{N}$. The inversion of $\Delta V_{T}$ is related with the large elastic deformation of the substrate in the perpendicular direction. Our results predict that the corrugation inversion occurs for substrates which have small $k_{z}$ for a wide range of perpendicular elastic deformation, and closely related with the second state in the stick-slip motion: For $F_{N}=0, F_{f}$ has welldefined value prior to the slip, and uniform profile but it exhibits anisotropy at the certain range of $F_{N}$. The range of $F_{N}$, where anisotropy in the stick-slip motion occurs, depends on the value of $k_{z}$. Upon the onset of anisotropy the stick-slip motion makes a transition from the first state $\left(\Delta V_{T}>0\right)$ to a second state $\left(\Delta V_{T}<0\right)$. The onset of second state prevented by the anharmonic and strongly repulsive forces opposing to the perpendicular deformation.

We finally consider the most fundamental aspect i.e the variation of $\bar{F}_{f}$ with $F_{N}$. This is usually taken $\bar{F}=\mu_{k} F_{N}$, which is true for the period of stick. In the range covering several stick and slip period the dynamic friction constant $\mu_{d}$ may not be constant. The relation between $\bar{F}_{f}$ and $F_{N}$ obtained depending on $k_{z}$ and anharmonicity of $V_{p, i}$ is illustrated in Fig. 5. For a surface which is rigid in the perpendicular direction $\left(k_{z} \rightarrow \infty\right) \mu_{d}$ is constant, but vary with $F_{N}$ when $k_{z}$ is finite. $\bar{F}_{f}$ decreases first, then increases with $F_{N}$ for $k_{z} \sim 10 \mathrm{eV} / \AA^{2}$. This behavior is related with the inversion of corrugation explained above. Depending upon the value of $k_{z}$ the minimum of the $\bar{F}_{f}\left(F_{N}\right)$ curve occurs at different $F_{N}$. 

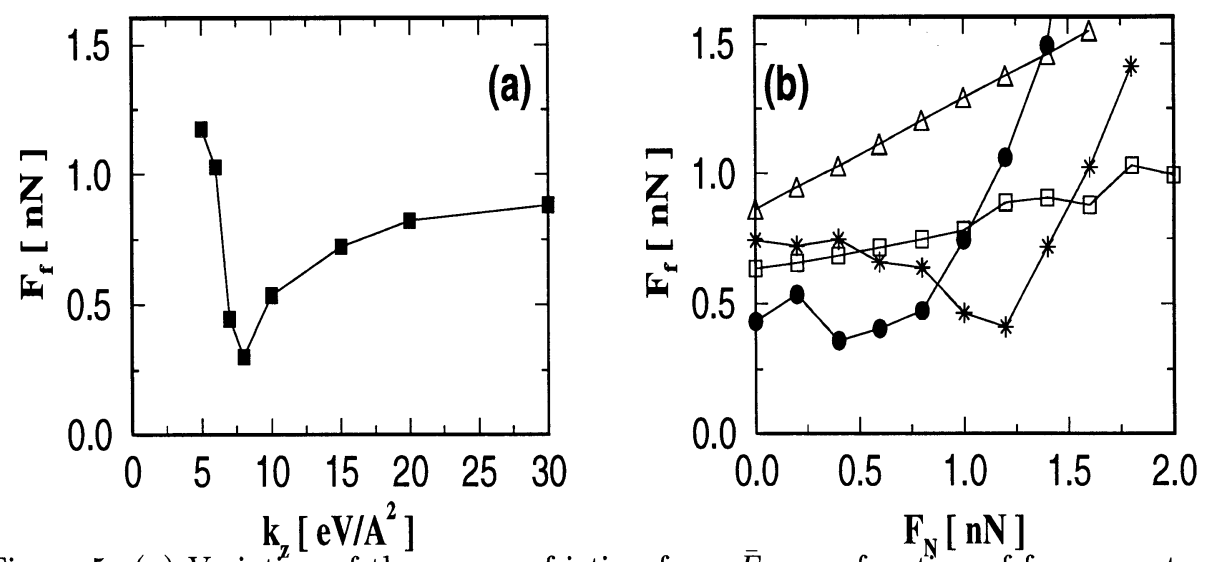

Figure 5: (a) Variation of the average friction force $\bar{F}_{f}$ as a function of force constant in the perpendicular direction $k_{z}$. (b) Average friction force versus normal loading for $F_{N}$ calculated for various $k_{z}$. Triangles, stars, circles and squares correspond to $k_{z}=\infty, 15,10$ $\mathrm{eV} / \AA^{2}$ and the case of anharmonic $V_{p, i}$, respectively.

For large $F_{N}$, the relation between $\bar{F}_{f}$ and $F_{N}$ becomes linear. At this point we examine the effect of anharmonic potential of the substrate atoms in the perpendicular direction, $V_{p, i}$. The potential including anharmonic contribution is expressed in terms of a polynomial up to $4^{\text {th }}$ power of the perpendicular displacement $\left(z_{i}-z_{i o}\right)$. For small displacements around the equilibrium position $V_{p, i} \sim k_{z}\left(z_{i}-z_{i o}\right)^{2} / 2$. Our calculations in the model described in Fig. 3 shows that the anisotropy of the stick-slip motion disappears gradually with increasing anharmonic contribution in $V_{p, i}$. As a result $\bar{F}_{f}\left(F_{N}\right)$ curve becomes linear for small loading force $F_{N}$. Nevertheless, the linear relation is destroyed again with increasing $F_{N}$.

\section{Boundary Lubrication}

In the presence of foreign atoms between flat metal surfaces the force between metal atoms of different surfaces is usually in the weak attractive range, while the force between metal and the adatom is in the (strong) repulsive range [24]. This way the foreign atoms prevent the surfaces from strong adhesion. The coverage of the foreign atom in the range $\Theta \sim 1$ is the boundary lubrication [15]. Some features of boundary lubrication are important for understanding of more complex lubrication. In this section we investigate the controlled motion of $\mathrm{Xe}$ on the $\mathrm{Pt}(111)$ surface [25]. The motion of Xe is induced by a moving object, which is taken to be the W-tip of STM. Such a model is convenient for several reasons: First one can establish direct connection with the experiment [26-27]. Second, since one moves only single Xe atom, the stick-slip motion of Xe can be easily analyzed. The tip moving on a metal surface is also relevant for a moving object having asperities. In comply with the discussion in Section 2 the interaction energy between xenon and the $\operatorname{Pt}(111)$ surface consists of i) short-range and attractive interaction energy due to the charge rearrangements in the chemical bond, ii) short-range repulsive energy, iii) long-range and attractive Van der Walls energy. It is usually argued that the charge rearrangement upon physisorption of closed-shell atoms, such as Xe on metal surfaces is negligible, and hence does not induce any short-range attractive interaction. On the contrary to this argument, the local den- 

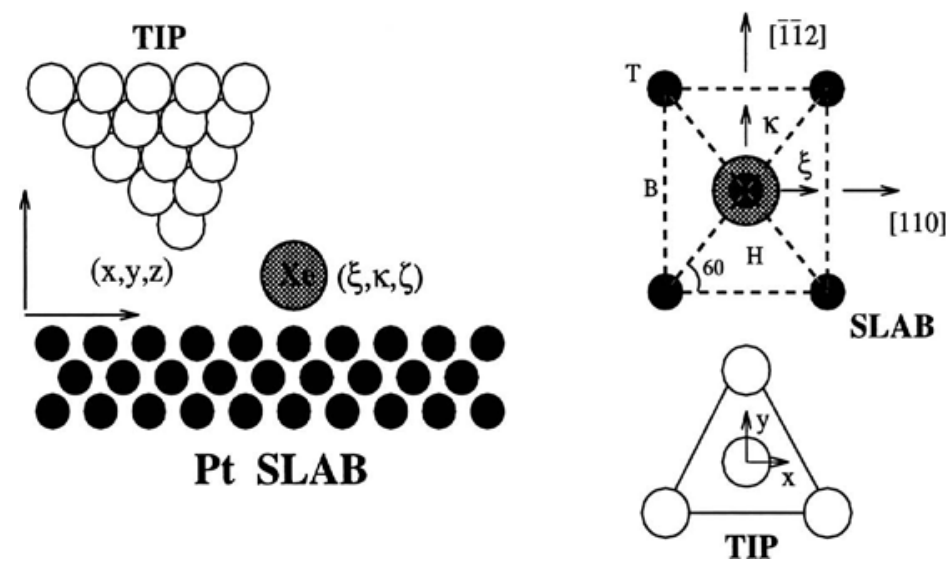

Figure 6: A schematic description of the STM tip, the Xe atom and the Pt(111) surface. The coordinates of the apex of the tip and the Xe atom are $(x, y, z)$ and $(\xi, \kappa, \zeta)$, respectively.

sity treatment of the interaction between close-shell atoms and jellium metal surfaces by Lang [28] provided a good account of experimental data on atomic binding energy, dipole moment and core-level binding energy shift. By examining work function measurements of rare gas atoms adsorbed on metal surfaces Ishi and Viswanathan [29] concluded that the chemical binding effects are essential in bonding. Recently, Baratoff et al [30] carried out self-consistent field pseudopotential calculation with the local density approximation for the binding energy of $\mathrm{Xe}$ on the $\mathrm{Al}(111)$ surface. The calculated binding energy was $130 \mathrm{meV}$, which is compared to the experimental binding energy of $200 \mathrm{meV}$. This indicates that even for the adsorbed closed-shell atoms the short-range interaction near the equilibrium distance dominates the weak long-range interaction. The free parameter LCAO calculation by Pérez et al [31]. also confirms this conclusion.

In the calculations [25] of lateral displacement, the $\mathrm{Pt}$ slab is fixed in the reference frame and is represented by $42 \mathrm{Pt}(111)$ atomic layers comprising $14112 \mathrm{Pt}$ atoms. The tip which represents the moving object is constructed by $2024 \mathrm{~W}$ atoms in pyramidal geometry generated from $22 \mathrm{~W}(111)$ layers. The apex of the tip has single atom; the second layer has three atoms. The coordinates of the tip (apex atom) and those of $\mathrm{Xe}$ relative to a point on the $\mathrm{Pt}$ surface are labeled by $(x, y, z)$ and $(\xi, \kappa, \zeta)$, respectively. The interaction potential between the Xe atom (at $\mathbf{R}_{\mathbf{a}}$ ) and any atom of the W-tip (at $\mathbf{R}_{\mathbf{l}}$ ) is expressed in terms of a Lennard-Jones pair potential having the well known form, $\epsilon\left[\left(r_{o} /\left|\mathbf{R}_{\mathbf{a}}-\mathbf{R}_{\mathbf{l}}\right|\right)^{12}-2\left(r_{o} /\left|\mathbf{R}_{\mathbf{a}}-\mathbf{R}_{\mathbf{l}}\right|\right)^{6}\right]$. The parameters of this potential [24] are determined from the experimental data to be $\epsilon=0.339 \mathrm{eV}$, and $r_{o}=3.62 \AA$. The many body effects were taken into account by scaling $\epsilon$ and $r_{o}$ values. Note that the Lennard-Jones pair potential function is only a crude approximation for the $\mathrm{Xe}-\mathrm{W}$ interaction; it could have improved by using additional terms. This, however, requires additional experimental data to fit, which are not available yet. The atomic arrangement of tip-adatom-Pt surface system and the orientation of the tip are schematically described in Fig.6.

The empirical potential introduced by Barker and Rettner [32] expresses the interaction 


\begin{tabular}{rrrr}
\hline & Binding Energy (meV) & Barrier Energy (meV) \\
\hline H-site & 222 & $\mathrm{~T} \rightarrow \mathrm{H} \rightarrow \mathrm{T}$ & 32 \\
T-site & 254 & $\mathrm{~T} \rightarrow \mathrm{B} \rightarrow \mathrm{T}$ & 26 \\
B-site & 228 & & \\
\hline
\end{tabular}

Table 1: Energetics of $\mathrm{Xe}$ on the $\mathrm{Pt}(111)$ surface. T,H,B denote for top, hollow and bridge sites, respectively.

between a single $\mathrm{Xe}$ atom and the $\mathrm{Pt}(111)$ surface in terms of the sum of non-spherical, pairwise additive potentials and of an additional term which describes the interaction of Xe with the delocalized conduction electrons of the sample surface. The nine parameters in the potential function were fitted to a wide range of available experimental data. Details of this potential can be obtained in Ref. 32 .

The objects [i.e the $\mathrm{W}$-tip and the $\mathrm{Pt}(111)$ slab] are taken rigid. As a result, the position of atoms in these objects are fixed at their bulk equilibrium positions and hence part of the interatomic interactions (such as $\mathrm{Pt}-\mathrm{Pt}, \mathrm{Pt}-\mathrm{W}$ and $\mathrm{W}-\mathrm{W}$ ) are not taken into account in the total potential function. The assumption of rigid objects is valid if the height $z$ of the tip is large. For relatively smaller tip-surface separations the modification of the atomic structure at the close proximity of the tip (such as elastic or plastic deformation, wetting and surface melting) have to be taken into account, however.

The potential energy surface of Xe is calculated at each grid point $(\xi, \kappa)$ on the $\operatorname{Pt}(111)$ surface by varying its height $\zeta$. Then, the potential energy surface is obtained by plotting the minimum values of the calculated energy at each grid point. Highest binding energy (or lowest potential energy) is $E_{b}=254 \mathrm{meV}$ and occurs at the top site. This is in contrast to many adsorbate-substrate systems, [ such as Xe on the $\mathrm{Al}(100)$ surface ] in which the hollow site occurs as the equilibrium binding site. The change of the adsorption site on the $\mathrm{Pt}$ surface is attributed to the reduction of $s+p$ electron density due to the $d$-states occupied near the Fermi level. The binding energy at the symmetry points of the unit cell and the energy barriers for different directions of motion are shown in Table I. The potential energy surface is strongly modified by a $\mathrm{W}$ tip approaching to the physisorbed Xe atom. The minimum of the potential energy, which is displaced towards the tip, usually follows the motion of the tip, if the tip-Pt surface distance is properly varied in the course of translation. The carriage of $\mathrm{Xe}$ on the $\operatorname{Pt}(111)$ surface as a function of the height of the tip $z$, is studied by performing molecular dynamics calculations. The results for the tip moving along the y-direction from a distance towards Xe at different heights $z$ are summarized in Fig.7. For $z=8 \AA$, the Xe atom practically remains unaffected. At $z=7.5 \AA$ the interaction between the tip and Xe becomes significant; first it is attracted by the motion of the tip, follows the tip for very short distance, but then it becomes unaffected by the tip. For $5 \AA<z<7 \AA$, $\mathrm{Xe}$ is attached to the tip and then is carried without showing any periodicity due to the surface corrugation. For this range of $z$ the interaction sets in already at $\kappa-y=5 \AA$ since the second layer of tip atoms are closer to $\mathrm{Xe}$; the $\mathrm{Xe}$ atom escapes $\sim 2.7 \AA$ sideways to the adjacent unit cell and at the same time is attached to the side face of the $\mathrm{W}$ tip. Only for smaller height of the tip $(z<5 \AA)$ the corrugation of the $\operatorname{Pt}(111)$ surface is reflected to the trajectory of $\mathrm{Xe}$. In this case, Xe escapes again sideways by $\sim 2.7 \AA$ to the adjacent top site and pushed by the tip moving along [112] direction. The jumps of the curve $d$ in 

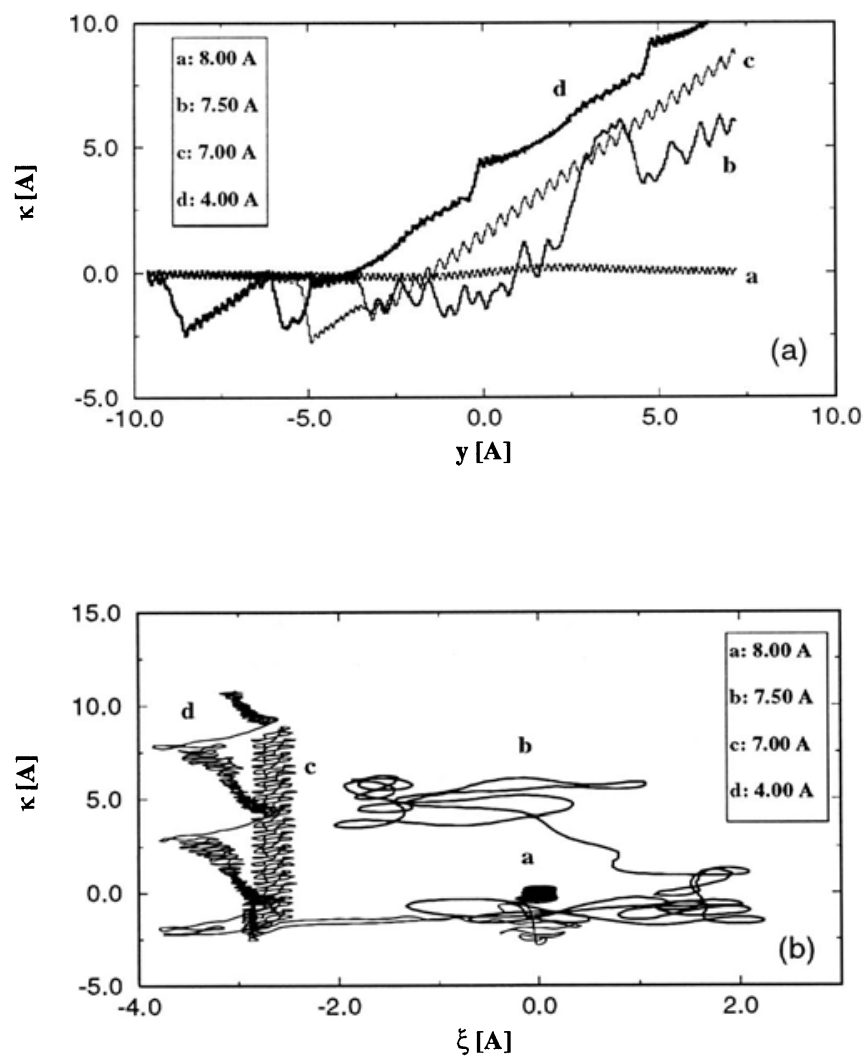

Figure 7: Lateral translation of $\mathrm{Xe}$ on the $\mathrm{Pt}(111)$ surface induced by the $\mathrm{W}$ tip moving along the $y$-[11ㅡㄹ direction. a,b,c and d correspond to different heights of the tip. Trajectories of Xe are shown in the $(\kappa, y)$ plane $(\mathrm{a})$; and $(\kappa, \xi)$ plane $(\mathrm{b})$.

the upper panel corresponds the Xe atom from one T-site to the next one along the [1112] direction. In the bottom panel of Fig. 7 we show the $(\kappa, \xi)$ trajectories of Xe corresponding to the same set of $z$ in panel (a). The Xe atom carried by the tip at $z=7 \AA$ traces a straight route along $\kappa$-axis with $\xi$ fixed at $\sim 2.7 \AA$. For $z=4 \AA$, the zig-zag trajectory is produced by $\mathrm{Xe}$, which is pushed from one T-site to the next one along the [1 $\overline{1} 2]$ direction by avoiding the $\mathrm{H}$-site. This behavior of pushing mode is rather different from that found in the controlled motion of $\mathrm{Xe}$ on $\mathrm{Ni}(110)$ surface [24].

The translation of $\mathrm{Xe}$ along the [110] direction (or $x$-direction) is similar to the one

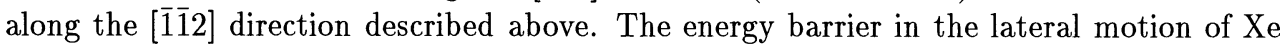
on the $\mathrm{Pt}(111)$ surface is smaller then that on the Ni(110) surface [26]. This is because the $\operatorname{Pt}(111)$ surface is closed-packed plane and the surface charge density has relatively smaller corrugation. Due to this fact the lateral translation of Xe on the surface exhibits the periodicity of the substrate only in certain range of $z$. The trajectories presented by Fig.7 are reminiscent of the stick-slip motion illustrated in Fig. 4. The trajectory corre- 
sponding to $z=4 \AA$ is relevant for the two-dimensional stick-slip motion of Xe. As the tip moves along the [11ㅡㄹ direction, the Xe atom traces zigzags over the adjacent top site and becomes attached to two T-sites in each period. Since the T-site is the binding (or lowest energy) site, the energy is stored while Xe is leaving one $\mathrm{T}$-site and is released suddenly approximately after the displacement of $\overline{T T} / 4$. This is the slip stage and energy is damped to local non-equilibrium phonons and electrons. Nevertheless, the energy damping is $s^{\prime \prime}$ expected smaller than the dry sliding friction since Xe-metal interaction is much smaller then metal-metal interaction energy.

\section{Conclusions}

In this work we study the dry sliding friction by using self-consistent field pseudopotential method with the local density approximation. The objects, which are taken to be rigid, are atomically flat. The effect of material parameters and loading force are investigated by using model calculations. We found that the elastic deformation in the perpendicular direction is important and gives rise to anisotropy in the stick-slip trajectories. Finally, the tip induced controlled motion of $\mathrm{Xe}$ on $\mathrm{Pt}(111)$ surface is studied. The stick-slip behavior is also observed in the motion of Xe on the metal surface. 


\section{References}

1. Bowden, F. P. and Tabor, D.(1965)Friction and Lubrication Methuen, London.

2. Rabinowicz, E. (1965) Friction and Wear Wiley, New York.

3. Israelachvili, J. N., McGuiggan, P.M. and Homola, H. M.(1987) Science 240, 189.

4. Grunze, M. and Kreuzer, H. J. (1989), Springer Series in Surface Sciences 17, Springer Verlag, Berlin; Bhushan, B., Israelachvili, J. N. and Landman, U.(1995) Nature 347, 607.

5. Binnig, G., Quate, C. F. and Gerber, Ch.(1986) Phys. Rev. Lett. 56, 930; Binnig, G., Gerber, Ch., Stoll, E., Albrecht, T. R. and Quate, C. F.(1987) Europhys. Lett. 3, 1281.

6. Mate, C. M., McClelland, G. M., Erlandsson, R. and Chiang, S.(1987) Phys. Rev. Lett. 59, 1942.

7. Meyer, E., Overney, R., Brodbeck, D., Howald, L., Lüth, R., Frommer, J. and Güntherodt, H. J.(1992) Phys. Rev. Lett. 69, 1777.

8. H. J. Günntherodt, D. Anselmetti and E. Meyer(eds.)(1995) Forces in Scanning Probe Methods Kluwer Academic Publishers, Dordrecht.

9. Marti, O.(1993) Surface and Coatings Technology 62510.

10. Overney, R. M., Takano, H., Fujihira, M., Paulus, W. and Ringsdorf, H.,(1994) Phys. Rev. Lett. 72, 3546.

11. Tomlinson, G. A.(1929) Philos. Mag. 7905.

12. Frenkel, J. and Kontorova, T.(1938) Phys. Z. Sowjetunion 13,1; Atkinson, W. and Cabrera, N.,(1965) Phys. Rev. 138, A763.

13. Sokoloff, J. B.(1991) Thin Solid Films 206, 208; (1993) Phys. Rev. B 47, 6106.

14. Tomanek, D., Zhong, W. and Thomas, H.(1991) Euorphys. Lett. 15, 887.

15. Persson, B. N. J.(1993) Phys. Rev. Lett. 71, 1212; (1994) Phys. Rev. B 50, 4771.

16. Gyalog, T., Bammerlin, M., Lüthi, R., Meyer, E. and Thomas, H.(1995) Europhys. Lett. 31, 269.

17. Landman, U., Luedtke, W. D. and Nitzan, A.(1989) Surf. Sci. 210, L177; Landman, U., Luedtke, W. D., Burnhan, N. A. and Colton, R. J.(1990) Science 248, 454. 
18. Thomson, P. A. and Robbins, M. O.(1990) Phys. Rev. A 41, 6830; (1990) Science 250, 792 .

19. Nieminen, J. A., Sutton, A. P. and Pethica, J. B.(1992) Acta. Met. 40, 2503;Nieminen, J. A., Sutton, A. P., Pethica, J. B. and Kaski, K.(1992) Modeling and Simulation in Materials Science and Engineering, 1, 83.

20. B. N. J. Persson and E. Tosatti (eds.), Physics of Sliding Friction, Nato ASI Ser. E, Vol. 286, (1996) Kluwer Academic Publishers, Dordrecht.

21. Matsukawa, H. and Fukuyama, H.,(1994) Phys. Rev. B 49, 17286.

22. Ciraci, S., Baratoff, A. and Batra, I. P.(1990) Phys. Rev. B 41, 2763; Ciraci, S.(1990) in J. Behm, H. Rohrer and N. Garcia(eds.), Basic Concepts and Applications of Scanning Tunneling Microscopy and Related Techniques, Kluwer Academic Publishers, Dordrecht.

23. Ciraci, S., Tekman, E., Baratoff, A. and Batra, I. P.(1992) Phys. Rev. B 46, 10411.

24. Buldum, A., Ciraci, S. and Erkoç, S.(1995), in H. J. Günntherodt, D. Anselmetti and E. Meyer(eds.)(1995) Forces in Scanning Probe Methods Kluwer Academic Publishers, Dordrecht; Buldum, A., Ciraci, S. and Erkoç, S.(1995), J. Phys. Condens. Matter 7, 8487.

25. Buldum, A. and Ciraci, S.(1996) Phys. Rev. B.

26. Eigler, D.M. and Schweizer, E. K.(1990) Nature 344, 524.

27. P. Avouris(ed.)(1993) Atomic and Nanometer-Scale Modification of Materials and Applications, Kluwer Academic Publishers, Dordrecht.

28. Lang, N. D.(1981) Phys. Rev. Lett. 46, 342.

29. Ishi, S. and Viswanathan, W.(1991) Thin Solid Fims 201, 373.

30. Baratoff, A., Ciraci, S. and Stoll, E.(unpublished).

31. Pérez, R., Garcia-Vidal, F. J., de Andrés P. L. and Flores, F.(1994) Surf. Sci307309, 704; Cerda, J. R., Flores, F., de Andrés P. L. and Echenique, P. M.(1993) Il Nuovo Cimento 15D, 451.

32. Barker, J. A., Rettner, C. T.(1992) J. Chem. Phys 97, 5844. 